\title{
Low Cost Adaptive Uterine Manipulator for Pelvic Laparoscopic Surgery
}

\author{
Antoine Edu ${ }^{1}$, Dan-Ioan Ulmeanu ${ }^{2}$, Petru-Lucian Milea ${ }^{3}$ \\ ${ }^{1}$ Amca Medical Srl, Matei Basarab 92B, Bucharest, Romania \\ 2"Titu Maiorescu" University, Faculty of Medicine, Gh. Petrascu 67A, Bucharest, Romania \\ ${ }^{3}$ POLITEHNICA University of Bucharest, Iuliu Maniu 1-3, Bucharest, Romania \\ ${ }^{1}$ simonaedu98@yahoo.com, ${ }^{2}$ d_ulmeanu@yahoo.com, ${ }^{3}$ lucian@intelprot.ro
}

\begin{abstract}
In this article we'll present an original uterine manipulator, very useful for pelvic surgery, especially for laparoscopic hysterectomies. There is a great diversity of uterine manipulators at present on the medical market, whichever making a manipulation of the uterus, a vaginal defining the seabed bag, ensuring the definition of anatomical elements for a quick and safe intervention. They can be for a single use or sterilizable, they have different degrees of constructive complexity and different reliability. Every manipulator type has specific advantages and disadvantages compared to others.
\end{abstract}

Our uterine manipulator has also some specific advantages over the manipulators we find on the market. These other uterine manipulators are briefly presented in the first section of the paper, for comparison reasons. Then our uterine manipulator and its parts are presented in details. Its advantages are: adaptive cup size and position, unbreakable, easy to clean and sterilize not conductive cup, increased reliability, low cost etc.

This original uterine manipulator was used in many types of pelvic and uterine surgery, where it proved to be very effective and reliable. The paper presents also some pictures taken from these surgeries.

Keywords: Uterine Manipulator, Pelvic Laparoscopic Surgery, hysterectomy, myomectomy. colpectomy

\section{INTRODUCTION}

Uterine manipulator is an indispensable device in laparoscopic gynecologic surgery in the pelvis involving easier and a more extensive mobilization of the uterus. Some interventions are inconceivable in the absence of this instrument. It ensures the mobilization of the uterus making it possible for operator to access anatomical structures to be treated during surgery (vascular pedicle, ligament, etc.). By manipulating the uterus, the operative field is exposed, thus, the more efficient the instrument in changing the position of the uterus, the faster and safer is the surgery.

\section{MATERIALS AND METHODS}

Laparoscopic surgeries in which the uterine manipulator is useful or even indispensable, are subtotal, total and debt widened hysterectomies, myomectomies, the surgery of pelvic endometriosis, the surgery regarding the restoration of pelvic statics and infertility surgeries.

The medical device market has a great diversity of uterine manipulators, most with a quite low margin of maneuverability, limited by the angle of the vagina, generally sterilizable but also for single use.

Advantages and disadvantages of each model, make the choice of the "ideal" manipulator very hard. Practical needs of operators encountered during gynecological interventions have led to progressive improvements of the instrument as well as increasing the versatibility - now a uterine manipulator can be used for many purposes related to pelvic laparoscopic surgery.

In our opinion, the most representative uterine manipulators types on the market are the following: 
a.Donnez type uterine manipulator (Fig.1.) designed specifically for total and subtotal hysterectomy. It doesn't allow the tip maneuverability in any plan [1].

b. Tintara type uterine manipulator (Fig.2.) - is an uterine manipulator that can be used for many laparoscopic gynecological indications, except for total hysterectomy. [1]

c. Clermont-Ferrand uterine manipulator (Fig.3.) is a uterine manipulator with multiple usages articulated, but through which contrast substance cannot be entered and has a traumatic top piece. [1]

d. Hohl uterine manipulator type - is a rigid instrument, used only for hysterectomies. [1]

e. Koninckx uterine manipulator type-is an articulated uterine manipulator, but traumatic, not allowing cromotubatia. [1]

f. Mangeshikar uterine manipulator type - is a uterine manipulator which fits directly into the uterine wall so it is traumatic. [1]

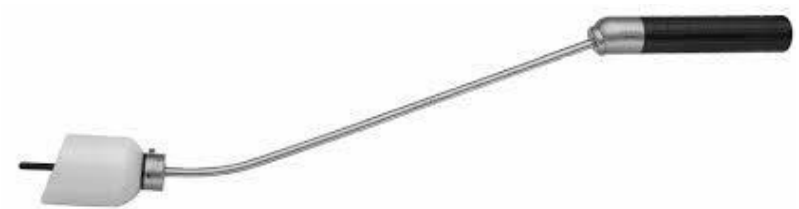

Fig. 1. Donnez type uterine manipulator [1]

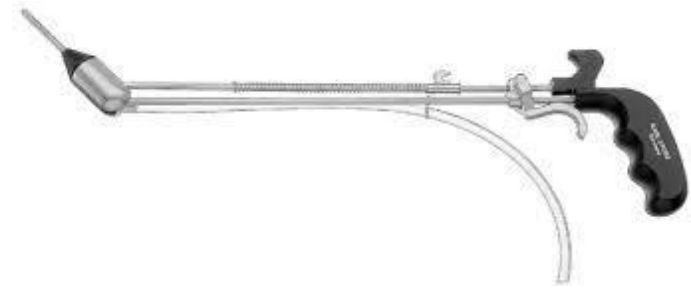

Fig. 2. Tintara type uterine manipulator [1]

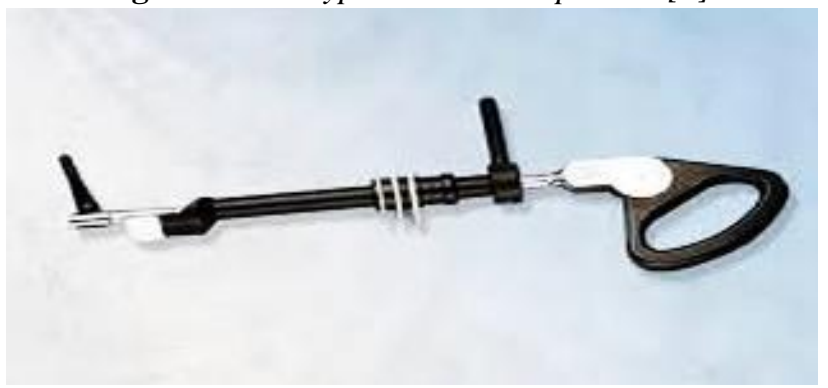

Fig. 3. Clermont-Ferrand type uterine manipulator [1]

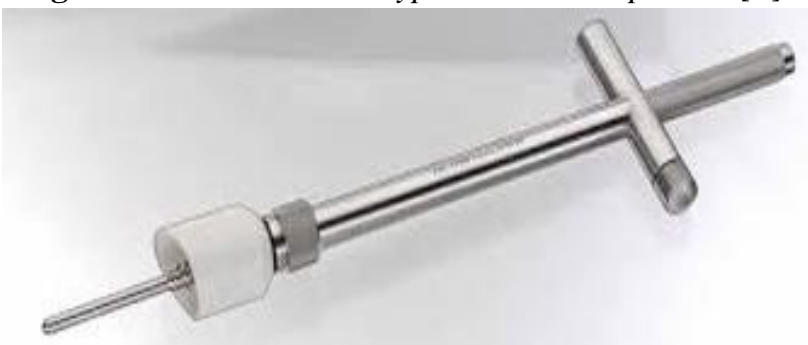

Fig. 4. Hohl type uterine manipulator [1]

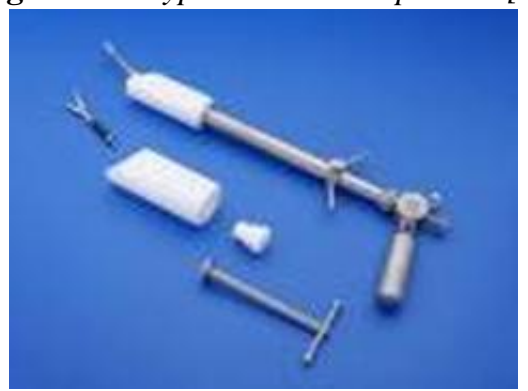

Fig. 5. Koninckx type uterine manipulator [1]

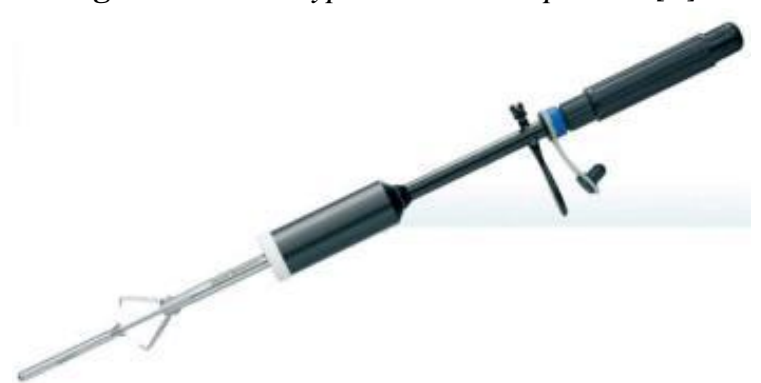

Fig. 6. Mangeshikar type uterine manipulator [1]

g. Tutunaru-Vârtej - Blowing uterine manipulator - consists, according to the invention [2], in a support rod provided at one end with a handle and at the other end with an inflatable; allows the mobilization of the uterus 
h. Advincula Arch type uterine manipulator radial, simple design with disposable parts. Allows to manually execute anteversion/ retroversion of the uterus. It permits easy insertion from different angles [3].

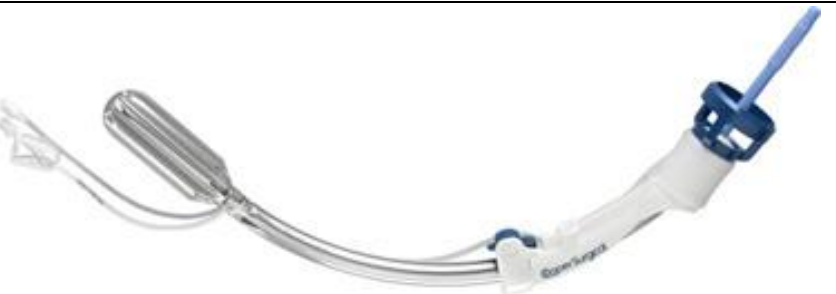

Fig. 7. Advincula Arch type uterine manipulator [3]

Manipulators characteristics are different depending on the model; the vast diversity suggests that is not yet an ideal tool currently on the market and there is room for improving the existing performance [4].

However, an articulated multifunctional uterine manipulator makes gynecologic surgery easier and safer. In the case of the articulated manipulator, the presentation of the operating field during hysterectomy or other conservative procedures it is much improved compared with conventional manipulators due to special configuration and its mobile parts [5].

Also, this tool does not injure tissues if using conservative surgery and allows tubal verifying through chromotubation. The cervix uterine pools with different sizes perfectly adapts to the anatomy, offering the operator multiple opportunities especially in difficult cases, is made of a material easily processed, mechanically resistant, sterilizable by approved methods, electrically unconductive and provides support for colpectomy.

Given the diversity of uterine manipulators on the market, one can ask legitimate - "can something be improved at this moment?", can we invent / imagine the "ideal" manipulator?

Our initial project falls into the category of relatively less maneuverable uterine manipulators, but it is very effective for specific tasks in pelvical surgery.

\section{Our Original Uterine Manipulator}

One of the most common types of pelvic surgery is the total laparoscopic hysterectomy. For this surgery we imagined and then built a dedicated instrument composed of two parts: a rod and a cup with sheath. The parts are presented below:

1.Rod Manipulator is very good fixed in the uterus, it basically screwing to the Uterine bottom (Fig.8). This is especially important for bulky fibroid uteri that ensure rigidity of the complex cervical - uterine body manipulation thus providing a better exposure of the slope posterior uterine broad ligament, uterosacral ligament.

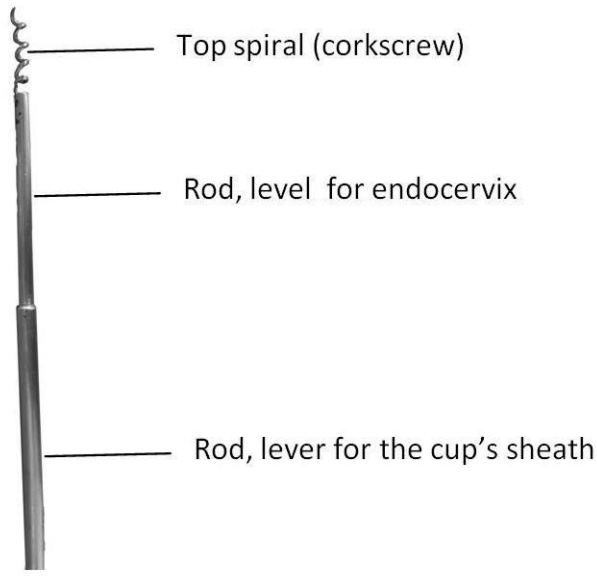

Fig 8. Rod manipulator

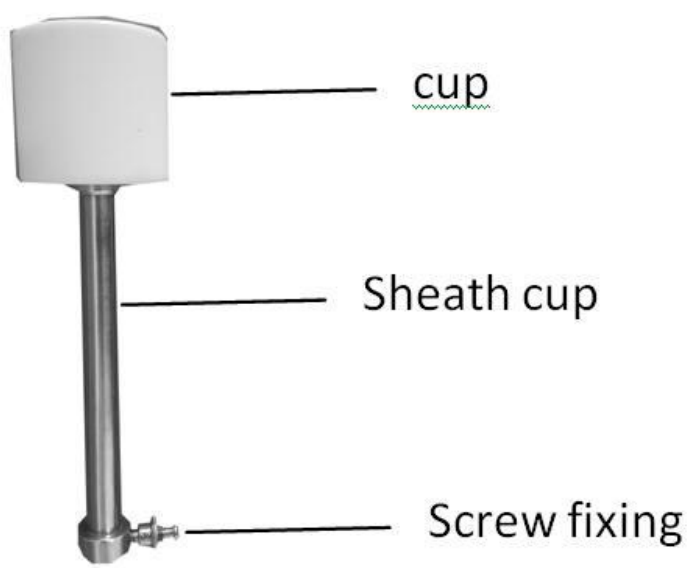

Fig 9. Cup

2.Cup (Fig.9). that defines Vaginal bag bottoms it is made of an unbreakable material, heat resistant, Insulating white in three dimensions (Fig.10) and can slide freely on the rod handler with the opportunity to be fixed at any time in the desired position related to the rod (Fig.11) 


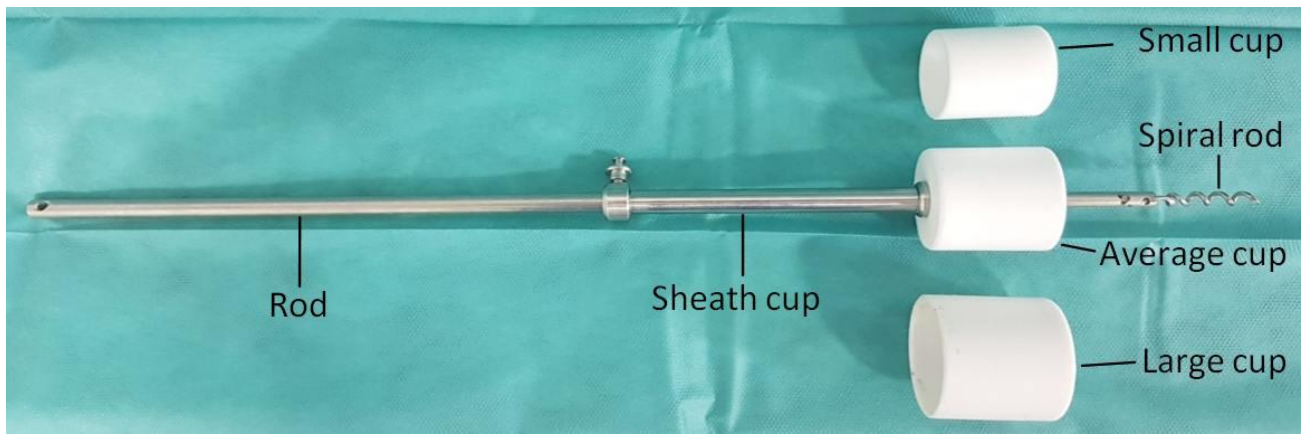

Fig 10. The manipulators with three cups' dimensions

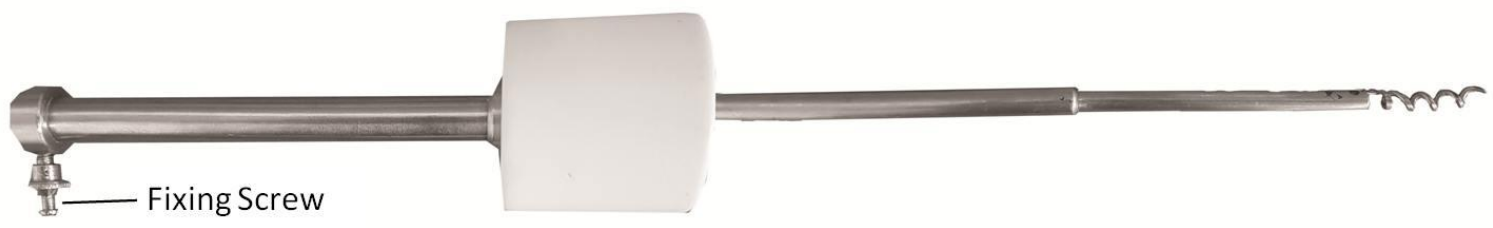

Fig 11. The assembled Instrument

The working technique of the manipulator is the following:

- Expose the cervix with valves

- Viewing cervical opening

- The hysterometry is made and the expansion needed until Hégar 6

- Under laparoscopic control, the manipulator rod is inserted until the top of helical (corkscrew) makes contact with the uterine wall background

The rod starts screwing clockwise positioning if needed the uterus in laparoscopy with forceps so that the effect of axialization and anteversion to be maximum. (Fig. 12 a)
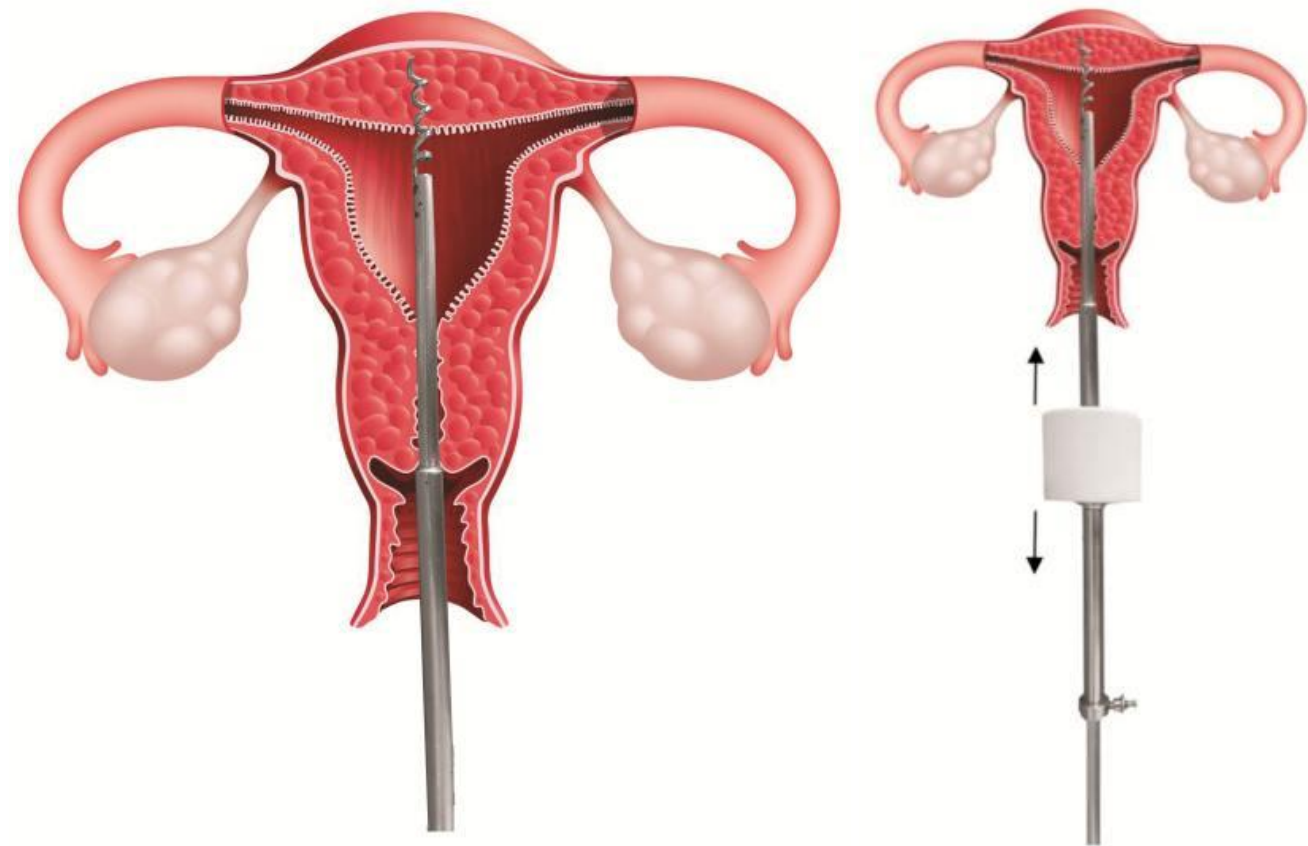

Fig.12. Rod screwed tight in the bottom of the myometrium: a) corkscrew fixing detail b) changing cup's position by sliding sheath on rod

The laparoscopic control allows the avoidance of the uterine perforation as the tip of the instrument approaches to the serous body producing a progressive local deformation, the progression rod ends and it can be removed by twisting $1 / 4-1 / 2$ turn counterclockwise. Once the rod is mounted the manipulator cup mounts dragging the cup's sheath along the rod (Fig.12 b). 
Keeping the rod firmly in place allows the reverse traction effect while the cup is pushed gently until the laparoscopic check demonstrates a good embossing of the bottom of the bag. Maintaining a slight tension between the rod (that is drawn) and cup (which is pushed) cup is fixed in the desired position by tightening the fixing screw (Figure 13).

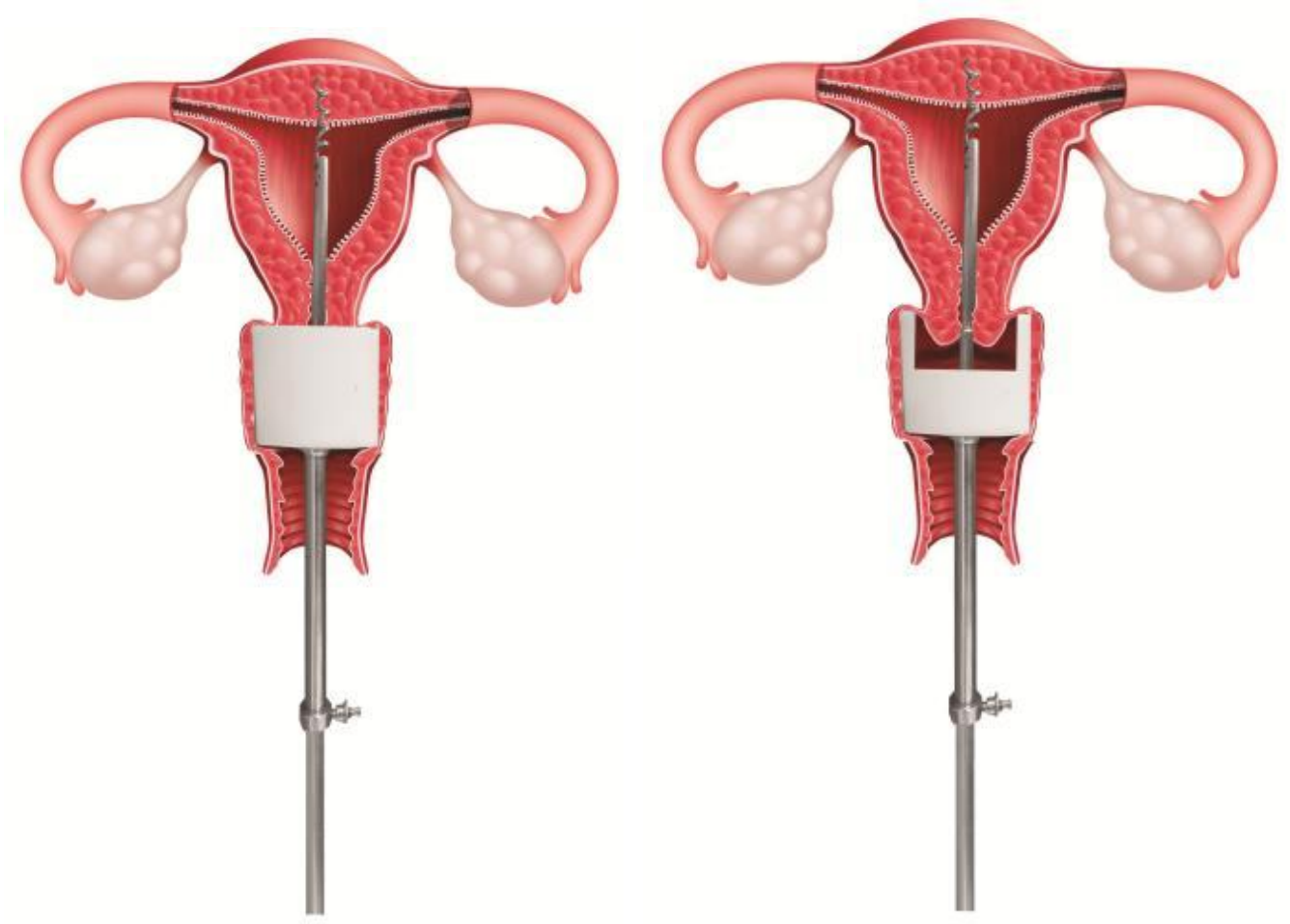

Fig13. Cup in final position, tightened by screw fixation: perspective (left) / section (right)

\section{RESULTS AND DISCUSSION}

We now have a solid overall fixing mechanical manipulator uterus leading to an efficient handling even on bulky uteri.

This is ensured by the particular elements of the proposed manipulator:

1. The rod with elicoidal peak

2. The cup with variable positioning and fixing related to the rod.

Taking into account the 3 cup sizes interchangeable, the instrument is suitable for a wide anatomical variety.

Most uterine manipulators have a rod entering through the cervical canal into the uterine cavity; on this rod a cup that can be fixed slides (pipe) in a certain predetermined position or the cup-rod assembly is fixed at the outset. The freedom of the rod, entering the uterine cavity has a fixed length, independent of the actual dimensions of the operated body.

For this reason in case of the bulky uteri it appears the "hinge" or "joint" effect because the part inside the uterus beyond the end of the rod is moving comparing to the riveted uterus because of the presence of the rod.

The presented uterine manipulator has a different mechanism of fixing:

- The rod that passes through the cervical canal and is fixed through the elicoidal top in the fundic uterine muscles, in all their thickness;

- At this point the rod is solidar with uterus and can be pushed along with it or hauled with it;

-The sliding of the cup is made simultaneously with a slight traction on the rod - so the uterus can be fully engagde in the cup;

- When the cup has ascended enough related to the rod, properly defining the bottom of the vaginal bag, the cup-rod assembly is fixed in this position. 


\section{Main Benefits:}

- The cup can be fixed at the desired level on the rod adapted to the real size of the operated body;

- Uterus-manipulator assembly is axially rigid disappearing the "hinge" or "joint" effect for bulky uteri. In addition the whole uterus-manipulator can be operated either by pushing (toward the peritoneal cavity) or tracting (toward the vagina).

\section{Other Advantages:}

- The cup is resistant, unbreakable, easy to clean and sterilize;

- Increasing reliability of assembly because of its constructive simplicity; the cup can be fixed and can slide easily on the rod, even after many uses (the cups with locking device have the disadvantage of fouling and clogging of this mechanism, while the cup no longer fixes right related to the rod);

- The manipulator is functional for different anatomical situations with varying distances between the bottom edge and uterine cervix so that the rod-cup assembly can be fixed in any desired position;

- Recovery is fast because achieving the surgery on a minimally invasive procedure; patients can leave the hospital after only one days after surgery, social and professional reinsertion is obviously improved compared to open surgery.

In laparoscopic radical gynecological operations (hysterectomy) the uterine manipulator is used both to mobilize in different positions of the uterine body and cutting the vagina (colpectomy). Below (Fig. $14)$, some image with manipulator's use in colpectomy are presented.
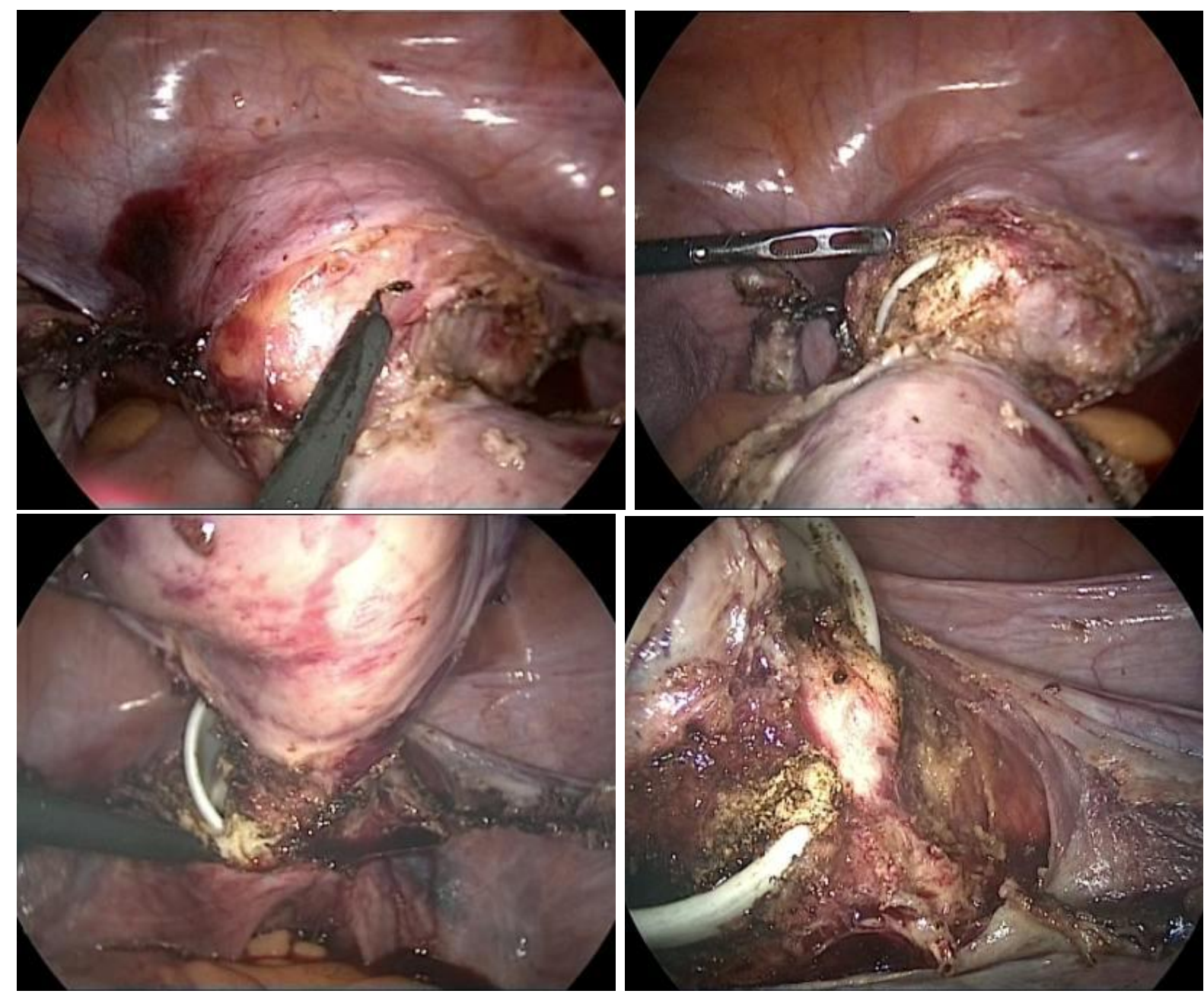

Fig 14. Colpectomy images: a) initiating the procedure b) manipulator's cup become visible c) back colpectomy continued d) almost complete colpectomy (the manipulator's cup can be observed)

The presented uterine manipulator was used in 358 laparoscopic surgeries, all claiming the being with favorable results. 


\section{Conclusions}

Using an uterine manipulator can simplify considerably the pelvic laparoscopic surgery. Complex surgical situations, deep infiltrative endometriosis or a large uterus often produce difficult access in the operative field.

The production of a uterine manipulator with a high degree of mobility lies in the need to expose certain areas with difficult access in the operating field for the surgeon.

The presented uterine manipulator has been used in over 350 laparoscopic surgeries, all with favorable results. It has significative advantages over the ones found on the market, like:

- Low price, below one quarter of the price of those on the market [1];

- Manipulator's cup can be fixed at any level on the rod and it's adapted to the actual dimensions of the body;

- The uterus-manipulator assembly is axially rigid, eliminating the "hinge" effect for bulky uteri;

- The cup is resistant, unbreakable, easy to clean and sterilize, electrically nonconductive (allowing the use of electric scalpel);

- Increased reliability due to the assembly's constructive simplicity; the cup can be fixed or slide easily on the rod, even after many uses (other devices on the market has cups with locking mechanisms which is fouling and clogging over many uses, while no longer fixes the cup right in relation to the rod);

- The manipulator is functional for different anatomical situations, with varying distances between the cervix extremity and uterine: the rod-cup assembly can be fixed in any desired position.

The presented tool is very effective but is dedicated only to surgical excision (hysterectomies, colpectomies etc.). But, there are many situations that require manipulation of the uterus without trauma to the uterine cavity or uterus in conservative procedures, so it is necessary to have a nontraumatic manipulator but with high handling performance, reliability, simple and versatile (enabling and injecting intrauterine colored substance for checking laparoscopic tubal patency or integrity of the uterine cavity after miometral suture (pelvic surgery for infertility, endometriosis, myomectomy) etc.

Such a non-traumatic uterine manipulator, with high degree of maneuverability, non-traumatic character, reliable, simple and versatile represent the wishes of a new type of uterine manipulator that will be subject to further research.

\section{ACKNOWLEDGMENTS}

The work presented in this paper was done using authors' private funding, but with institutional support from employing institutions.

\section{REFERENCES}

[1] Karl Storz, The World of Endoscopy - KARL STORZ - Tradition, Innovation, Expertise, 7th Edition 1/2015, https://www.karlstorz.com

[2] D. Tutunaru, P. Vârtej, Manipulator Uterin, Patent A61B1/303, OSIM, http://www.osim.ro/publicatii/brevete/bopi_2000/bopi1200.pdf

[3] CooperSurgical, RUMI2 System - Advanced Uterine Manipulation System, 2014, t www.coopersurgical.com

[4] L. van den Haak, C. Alleblas, T. E. Nieboer, J. P. Rhemrev, F. W. Jansen, Efficacy and safety of uterine manipulators in laparoscopic surgery: a review, Archives of Gynecology and Obstetrics, November 2015, Volume 292, Issue 5, pp 1003-1011, April 2015, DOI: 10.1007/s00404-0153727-9

[5] L. Mettler, Y.A. Nikam, A comparative survey of various uterine manipulators used in operative laparoscopy, Gynecol Surg (2006) 3: 239-243, DOI 10.1007/s10397-006-0215-z 